Science Signaling

MAAAS
Macromolecular assembly of the adaptor SLP-65 at intracellular vesicles in resting $B$ cells

Michael Engelke, Sona Pirkuliyeva, Julius Kühn, Leo Wong, Janina Boyken, Nadine Herrmann, Stefan Becker, Christian Griesinger and Jürgen Wienands (August 19, 2014)

Science Signaling 7 (339), ra79. [doi:

10.1126/scitranslmed.2005104]

The following resources related to this article are available online at http://stke.sciencemag.org.

This information is current as of September 29, 2014.

Article Tools Visit the online version of this article to access the personalization and article tools:

http://stke.sciencemag.org/content/7/339/ra79

\section{Supplemental}

Materials

Related Content

\author{
References
}

Glossary

Permissions
"Supplementary Materials"

http://stke.sciencemag.org/content/suppl/2014/08/15/7.339.ra79.DC1.html

The editors suggest related resources on Science's sites: http://stke.sciencemag.org/content/sigtrans/6/297/ra91.full.html http://stke.sciencemag.org/content/sigtrans/5/235/ra54.full.html http://stke.sciencemag.org/content/sigtrans/7/343/ra89.full.html

This article cites 33 articles, 11 of which you can access for free at: http://stke.sciencemag.org/content/7/339/ra79\#BIBL

Look up definitions for abbreviations and terms found in this article: http://stke.sciencemag.org/cgi/glossarylookup

Obtain information about reproducing this article: http://www.sciencemag.org/about/permissions.dtl

Science Signaling (ISSN 1937-9145) is published weekly, except the last December, by the American Association for the Advancement of Science, 1200 New York Avenue, NW, Washington, DC 20005. Copyright 2014 by the American Association for the Advancement of Science; all rights reserved. 


\title{
Macromolecular assembly of the adaptor SLP-65 at intracellular vesicles in resting $B$ cells
}

\author{
Michael Engelke, ${ }^{1}$ Sona Pirkuliyeva, ${ }^{1 \star}$ Julius Kühn, ${ }^{1 \star}$ Leo Wong, ${ }^{2}$ Janina Boyken, ${ }^{3 \dagger}$ \\ Nadine Herrmann, ${ }^{1}$ Stefan Becker, ${ }^{2}$ Christian Griesinger, ${ }^{2}$ Jürgen Wienands ${ }^{1 \ddagger}$
}

\begin{abstract}
The traditional view of how intracellular effector proteins are recruited to the B cell antigen receptor (BCR) complex at the plasma membrane is based on the occurrence of direct protein-protein interactions, as exemplified by the recruitment of the tyrosine kinase Syk (spleen tyrosine kinase) to phosphorylated motifs in BCR signaling subunits. By contrast, the subcellular targeting of the cytosolic adaptor protein SLP-65 (Src homology 2 domain-containing leukocyte adaptor protein of $65 \mathrm{kD}$ ), which serves as a proximal Syk substrate, is unclear. We showed that SLP-65 activation required its association at vesicular compartments in resting $B$ cells. A module of $\sim 50$ amino acid residues located at the amino terminus of SLP-65 anchored SLP-65 to the vesicles. Nuclear magnetic resonance spectroscopy showed that the SLP-65 amino terminus was structurally disordered in solution, but could bind in a structured manner to noncharged lipid components of cellular membranes. Our finding that preformed vesicular signaling scaffolds are required for B cell activation indicates that vesicles may deliver preassembled signaling cargo to sites of BCR activation.
\end{abstract}

\section{INTRODUCTION}

Various modes of direct protein-protein interactions play central roles in coupling the ligated $\mathrm{B}$ cell antigen receptor $(\mathrm{BCR})$ to its downstream signal effectors. Most notable are the inducible and constitutive associations mediated by Src homology (SH) 2 and 3 domains, respectively (1). The tandem SH2 domains of the tyrosine kinase Syk (spleen tyrosine kinase) bind to doubly phosphorylated versions of the immunoreceptor tyrosinebased activation motifs (ITAMs) in the BCR signaling subunits immunoglobulin $\alpha$ (Ig- $\alpha)($ CD79a) and Ig- $\beta$ (CD79b) (2). This arrangement equips the activated BCR with enzymatic activity. Similarly, the SH2 domaincontaining leukocyte adaptor protein of $65 \mathrm{kD}$ (SLP-65) (3), which is also called BLNK (4), has a distinct phosphotyrosine-based docking site in Ig- $\alpha$ $(5,6)$. The binding of SLP-65 to Ig- $\alpha$ facilitates Syk-mediated phosphorylation of SLP-65, which is instrumental for the activation of a plethora of B cell signaling cascades and, hence, humoral immunity in mouse and humans $(7,8)$. Moreover, the SH2 domain of SLP-65 can bind to phosphorylated Syk directly (9).

In addition to these inducible protein-protein interactions, SLP-65 also requires a constitutive association with Cbl-interacting protein of $85 \mathrm{kD}$ (CIN85) to translocate to the plasma membrane, and for the concomitant triggering of B cell activation pathways (10). Permanent complex formation between SLP-65 and CIN85 involves three proline-rich motifs in the central part of SLP-65 and the three N-terminal SH3 domains of CIN85. Exactly how CIN85 controls the subcellular movement of SLP-65 is unclear; however, the SLP-65 recruitment process appears to be even more complex. Mutational analyses showed that a short N-terminal SLP-65 segment of about 50 amino acid residues is indispensable for SLP-65 signaling (11). Furthermore, deletion of the N terminus of SLP-65 inter-

\footnotetext{
${ }^{1}$ Institute of Cellular and Molecular Immunology, Georg August University of Göttingen, Humboldtallee 34, 37073 Göttingen, Germany. ${ }^{2}$ Department of NMR-based Structural Biology, Max Planck Institute for Biophysical Chemistry, Am Fassberg 11, 37077 Göttingen, Germany. ${ }^{3}$ Department of Neurobiology, Max Planck Institute for Biophysical Chemistry, 37077 Göttingen, Germany. *These authors contributed equally to this work. †Present address: Bayer Health Care, Müllerstraße 178, Berlin, Germany ‡Corresponding author. E-mail: jwienan@gwdg.de
}

fered neither with the functionality of the SLP-65 SH2 domain nor with the ability of SLP-65 to form a complex with CIN85 $(12,13)$. These findings demonstrated that, in addition to the SH2 domain and the CIN85binding sites, the N-terminal region of SLP-65 constitutes a third and separate targeting device that controls a nonredundant route of SLP-65 activation.

The mode of action of the N terminus of SLP-65 remains unclear. It is characterized by a large number of basic and aliphatic amino acid residues. A putative leucine zipper motif was predicted to recognize a ligand leucine zipper in membrane-targeting proteins (11). The elucidation of the SLP-65 interactome by SILAC (stable isotope labeling by amino acids in cell culture)-based quantitative mass spectrometry did not, however, reveal a protein ligand for a putative leucine zipper or any other part of the $\mathrm{N}$ terminus of SLP-65 (13). Here, we showed that the $\mathrm{N}$ terminus of SLP-65 mediated direct protein-lipid interactions. Unexpectedly, the lipid-binding activity of SLP-65 was required for B cell activation before BCR stimulation occurred to position SLP-65 in cooperation with CIN85 at small, exosome-like intracellular compartments. Our data suggest that the ability of SLP-65 to respond to BCR activation requires its stimulationindependent residence at vesicular compartments that may en masse deliver a preformed cargo of signaling effector molecules to sites of BCR activation. This process would provide an efficient mechanism to amplify signal initiation and processing at activated receptors.

\section{RESULTS}

\section{Signaling-competent SLP-65 resides in particulate cell compartments}

Köhler et al. and our own group previously showed that the $\mathrm{N}$ terminus of SLP-65 is essential to receive and process signals from the activated BCR (fig. S1) $(11,12)$. To investigate the underlying mechanism, we visualized the subcellular distribution of citrine-tagged versions of wild-type and N-terminally truncated SLP-65 ( $\Delta \mathrm{N}-\mathrm{SLP}-65)$ with laser scanning confocal microscopic analysis of resting and BCR-activated transductants of the chicken B cell line DT40 (Fig. 1A). In the absence of BCR stimulation, wild-type SLP-65 was localized in intracellular speckles that were 
distributed throughout the cytoplasm (Fig. 1A). These dot-like structures entirely disappeared $1 \mathrm{~min}$ after BCR engagement (Fig. 1A). Instead, SLP-65-derived fluorescent signals were detected at the plasma mem-

A

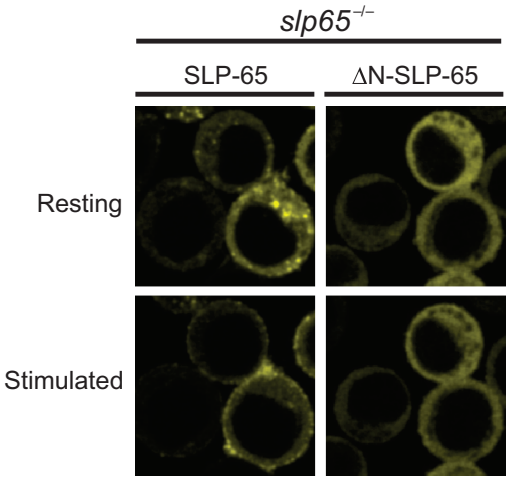

C

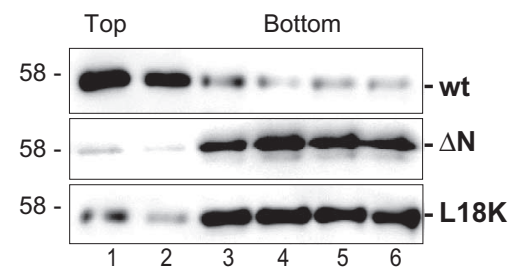

E Liposome added: $\nrightarrow 3.9 \mathrm{mM} \rightarrow 7.8 \mathrm{mM} \multimap 10.9 \mathrm{mM}$ GSNKITVPASOKLROLOKMVHDIKNNEGGIMNKIKKLKVKAPPSVPRR

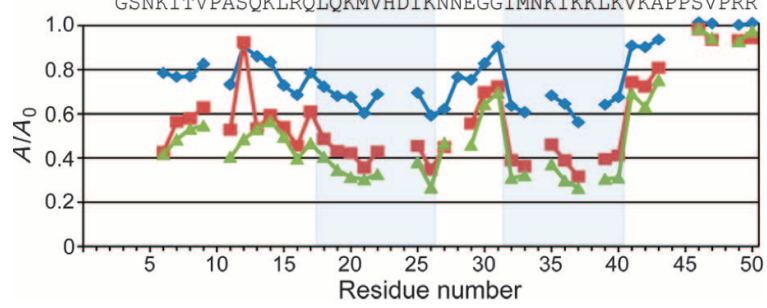

Fig. 1. The SLP-65 $N$ terminus associates with intracellular membranes. (A) SLP-65-deficient DT40 cells reconstituted with citrine-tagged wild-type (wt) SLP-65 (left) or $\Delta$ N-SLP-65 (right) were analyzed by confocal laser scanning microscopy. Images from cells before (resting) or after stimulation through their BCR with anti-IgM antibodies for $1 \mathrm{~min}$ are representative of 20 experiments. (B) DT40 cells expressing wt SLP-65 (top) or the signalingincompetent variants $\Delta \mathrm{N}$-SLP-65 or L18K-SLP-65 (second and third panels, respectively) were mechanically homogenized and subjected to ultracentrifugation on a sucrose step gradient of the indicated concentrations (SC). Individual fractions were analyzed by Western blotting with antibodies against SLP-65 (top three panels) or Akt1 (bottom), which is a cytosolic marker. IP, interphase. Western blots are representative of three independent experiments. The positions of molecular mass markers $(\mathrm{kD})$ are indicated on the left. (C) Recombinant wt SLP-65, $\Delta N-S L P-65$, and L18K-SLP-65 proteins were incubated with SUVs composed of $75 \%$ phosphatidylcholine, 23\% phosphatidylethanolamine, and 2\% Texas Red-labeled phosphatidylethanolamine (as tracer). Protein-liposome mixtures were loaded onto a Nycodenz gradient and subjected to ultracentrifugation, whereupon SUVs and potentially bound proteins floated to the two topmost fractions (1 and 2). Individual fractions were collected, and their protein brane, reflecting the BCR-induced translocation of SLP-65. Unlike wild-type SLP-65, the $\triangle \mathrm{N}-\mathrm{SLP}-65$ variant was almost uniformly dispersed in the cytoplasm of both unstimulated and stimulated B cells

B

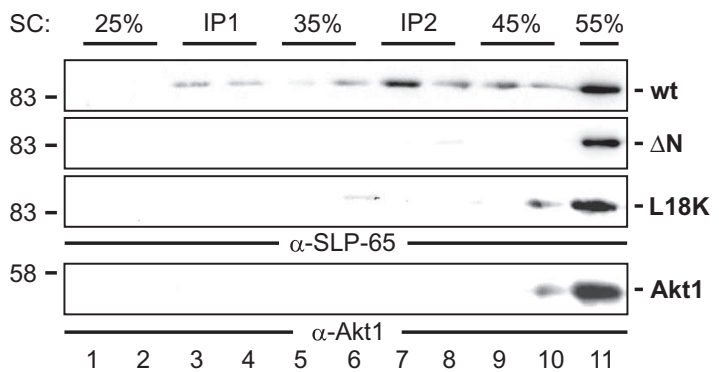

D

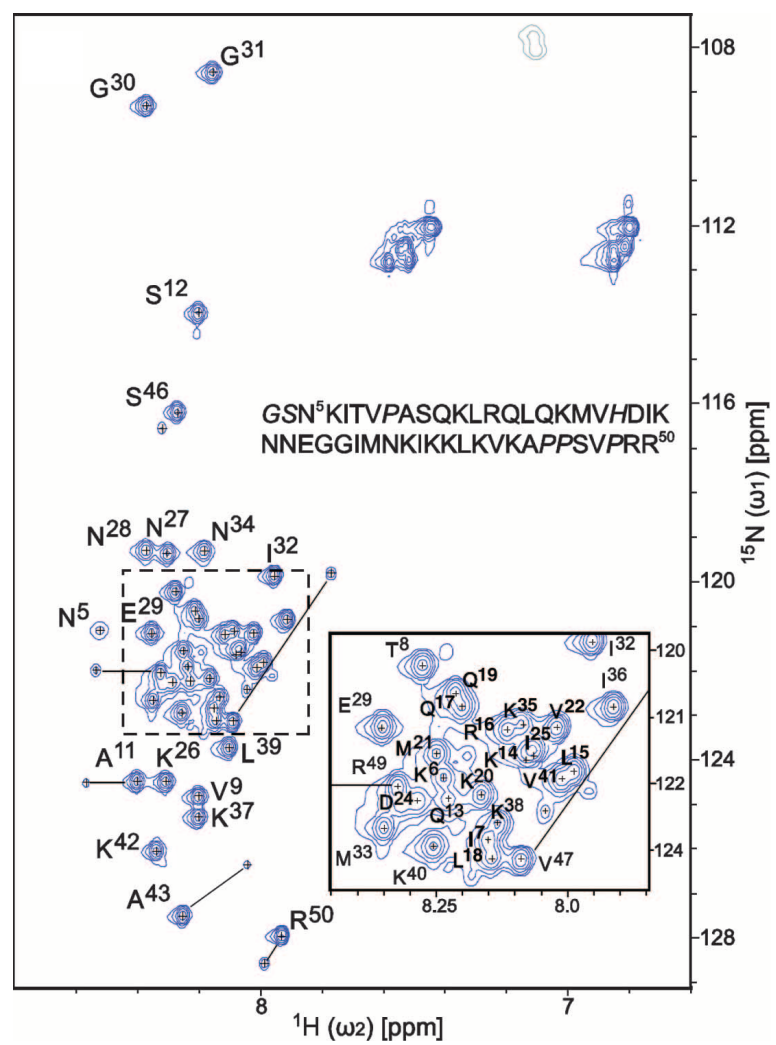

distributions were determined by Western blotting analysis with anti-SLP-65 antibodies. Western blots are representative of three independent experiments. (D) ${ }^{15} \mathrm{~N}-\mathrm{HSQC}$ (heteronuclear single quantum correlation) spectrum of wt SLP-65 (amino acid residues 5 to 50 ) at $25^{\circ} \mathrm{C}$. Residues adjacent to proline residues appear as a major and a minor peak, indicating the existence of cis-trans isomers of the proline residues. The chemical shift dispersion of the backbone amides is about 1 part per million (ppm), which is compatible with the intrinsically disordered conformation of the $\mathrm{N}$ terminus in aqueous solution. (E) Differential attenuation of the peak amplitude $\left(A / A_{0}\right)$ in ${ }^{15} \mathrm{~N}-\mathrm{HSQC}$ of wt SLP-65 (amino acid residues 5 to 50 ) was observed (see fig. S4C) upon titration with increasing amounts of liposomes, as indicated. Residues with low $A / A_{0}$ values reflect the vicinity of liposome-binding sites. 
(Fig. 1A). These data confirmed the importance of the $\mathrm{N}$ terminus for the relocalization of SLP-65 upon BCR activation, and moreover revealed that the N terminus controls SLP-65 localization even in resting B cells.

The dot-like appearance of SLP-65 could be explained by multimerization of wild-type SLP-65 or by its residence in defined subcellular compartments. We could not coimmunoprecipitate differently tagged versions of SLP-65 from transduced B cells, which suggested that direct oligomerization of SLP-65 was unlikely (fig. S2). We thus established a Balch homogenizer-based cell fractionation assay that enabled us to disrupt $\mathrm{B}$ cells in the absence of detergent and, hence, to preserve membranous cell compartments that could be further separated from the soluble molecules of the cytosol by ultracentrifugation on a sucrose gradient. In this setting, particulate cell fractions floated to the interphases of the sucrose gradient, whereas soluble molecules of the cytosol remained at the bottom of the centrifugation tube. Subsequently, individual cell fractions were subjected to Western blotting analysis with antibodies specific for SLP-65 or the serine and threonine kinase Akt, which served as a marker for cytosolic proteins (Fig. 1B). In contrast to Akt, signaling-competent wild-type SLP-65 of chicken and human B cells was found in the cytosolic fraction and in interphase 2 (Fig. 1B and fig. S3). Interphase 2 accommodated cell compartments that were of a higher density than those that migrated in interphase 1, which contained only minute amounts of wild-type SLP65 (Fig. 1B). By contrast, $\Delta \mathrm{N}-\mathrm{SLP}-65$ was exclusively found in the cytosolic fraction (Fig. 1B). Likewise, a signaling-incompetent point mutant of SLP-65 containing a leucine-to-lysine substitution at position 18 (L18K) was purely cytosolic (Fig. 1B). These data show that the presence of a functional $\mathrm{N}$ terminus is associated with the ability of SLP-65 to reside in particulate cell fractions. Note that not only deletion of the entire $\mathrm{N}$ terminus of SLP- 65 but also substitution of a single amino acid residue that preserves the overall positive charge of the $\mathrm{N}$ terminus blocked all three aspects of SLP-65 function: signal-independent localization to noncytosolic compartments, inducible translocation to the plasma membrane, and signaling (fig. S4).

\section{The N-terminal segment of SLP-65 constitutes a membrane-binding module}

Next, we performed an in vitro floatation assay to investigate whether the $\mathrm{N}$ terminus targeted SLP-65 to membrane compartments directly or whether this occurred indirectly because of an association between the SLP-65 N terminus and a protein ligand that itself was attached to or integrated into cellular membranes. We therefore prepared small unilamellar vesicles (SUVs) composed of 75\% phosphatidylcholine and $25 \%$ phosphatidylethanolamine and incubated them with recombinant wild-type SLP-65 protein or its N-terminal mutants, $\triangle \mathrm{N}-\mathrm{SLP}-65$ and L18K-SLP-65. After ultracentrifugation on a Nycodenz gradient, SUVs and potentially bound proteins floated to the top of the tube, whereas soluble proteins became gradually distributed in the tube. Individual fractions were analyzed by Western blotting with an anti-SLP-65 antibody as described earlier (Fig. 1C). Wild-type SLP-65 was concentrated in the SUV fractions and was almost absent in the soluble fractions. Conversely, none of the two signaling-incompetent SLP-65 mutants floated with the SUVs, but remained soluble (Fig. 1C). Hence, these data suggest that the N terminus of SLP-65 is capable of directly binding to general components of cellular membranes.

This result was further corroborated by nuclear magnetic resonance (NMR) spectroscopy, which demonstrated that the isolated SLP-65 N terminus was intrinsically disordered in solution (Fig. 1D and fig. S5) as inferred from calculations based on the chemical shifts of backbone amides, $\mathrm{C} \alpha$ and $\mathrm{C} \beta$ resonances (14). NMR spectroscopy also confirmed that the $\mathrm{N}$ terminus associated directly with liposomes (Fig. 1E and fig. S5). The formation of a helical structure upon liposome binding is likely given that hydrophobic residues appear every 3.5 residues in the membranebinding N-terminal segment. Moreover, this analysis identified individual amino acid residues that were in direct contact with the membrane (Fig. 1E). These were mainly hydrophobic and basic residues and included Leu ${ }^{18}$, which was identified by our mutational analysis to be indispensable for SLP-65 signaling. In addition, those aliphatic residues of the predicted leucine zipper (11) were tightly involved in SUV binding. However, inactivation of the motif by exchange of the central $\mathrm{Ile}^{25}$ for other aliphatic residues, such as alanine, which preserves interactions with hydrophobic membranes, or lysine, which maintains the basic nature of the $\mathrm{N}$ terminus, only marginally impaired SLP-65-induced $\mathrm{Ca}^{2+}$ signaling (fig. S6). Our data excluded the possibility of a functional leucine zipper motif and suggest that the $\mathrm{N}$ terminus confers signaling competence to SLP-65 by tethering this BCR effector directly to intracellular membranes. Hence, the N terminus-dependent dot-like structures of SLP-65 observed in B cells (Fig. 1A) appear to represent macromolecular assemblies of signaling-competent SLP-65 at intracellular vesicular compartments.

\section{SLP-65-positive vesicles accommodate exosomal marker proteins}

To further characterize vesicular SLP-65 in the cell, we used confocal microscopy to monitor the potential colocalization of citrine-tagged wildtype SLP-65 with various cerulean-tagged vesicular marker proteins that are characteristic for compartments of the trans-Golgi network or the endocytic and exocytic pathways in resting DT40 cells (Fig. 2A). No colocalization of SLP-65 was observed with the trans-Golgi network marker TGN38 or with a cerulean-labeled fusion protein that encompasses the pleckstrin homology $(\mathrm{PH})$ domain of oxysterole-binding protein 1 (OSBP1). The OSBP1-PH domain binds with distinct specificity to phosphatidylinositol4-monophosphate (PIP4), which resides exclusively in Golgi-proximal vesicles (15). Similarly, labeling of vesicles with cerulean-tagged Rab proteins that are specific for endosomes, with the soluble $N$-ethylmaleimidesensitive protein attachment protein receptor (SNARE) family member SNAP23, or with syntaxin 8 did not reveal colocalization with SLP-65 (Fig. 2A and fig. S7). SLP-65 was also absent from Rab8- and Rab27positive exosomal compartments (Fig. 2A).

We observed marked colocalization of SLP-65 with VAMP7 (vesicleassociated membrane protein 7), a member of the family of SNARE molecules that is mainly located at exosomes (16), and with quinacrine, which stains ATP (adenosine 5'-triphosphate)-containing exosomes (Fig. 2A) (17). Quantification of the colocalization confirmed the specific residence of wild-type SLP-65 at this distinct species of exosome-like vesicles in resting B cells (Fig. 2B). Further evidence for the $\mathrm{N}$ terminus-dependent attachment of SLP-65 to VAMP7-positive vesicles was obtained by a bimolecular fluorescence complementation assay in which the two proteins were fused to either the $\mathrm{N}$ - or the $\mathrm{C}$-terminal parts of the Venus fluorophore, respectively. In transduced B cells that contained both fusion proteins, the two portions of Venus could combine to complement the fluorescence signal (fig. S8). No signal was observed in cells containing Venus-tagged $\Delta$ N-SLP-65 (fig. S8). However, the vesicular colocalization of SLP-65 and VAMP7 does not necessarily imply that the two proteins are structurally linked or that VAMP7 directly contributes to SLP-65 signaling. Indeed, fluorescence recovery after photobleaching (FRAP) analysis revealed that the vesicular pool of SLP-65, but not VAMP7, rapidly exchanged with the cytosolic pool (fig. S9). The latter result also excluded the possibility that SLP-65 was located inside the vesicles. Together, our findings suggest that the ability of SLP-65 to receive and process upstream activation signals depends on its ability to assemble at vesicular platforms before BCR stimulation occurs. The exact composition and 
A TGN38

OSBP1-PH

Rab5
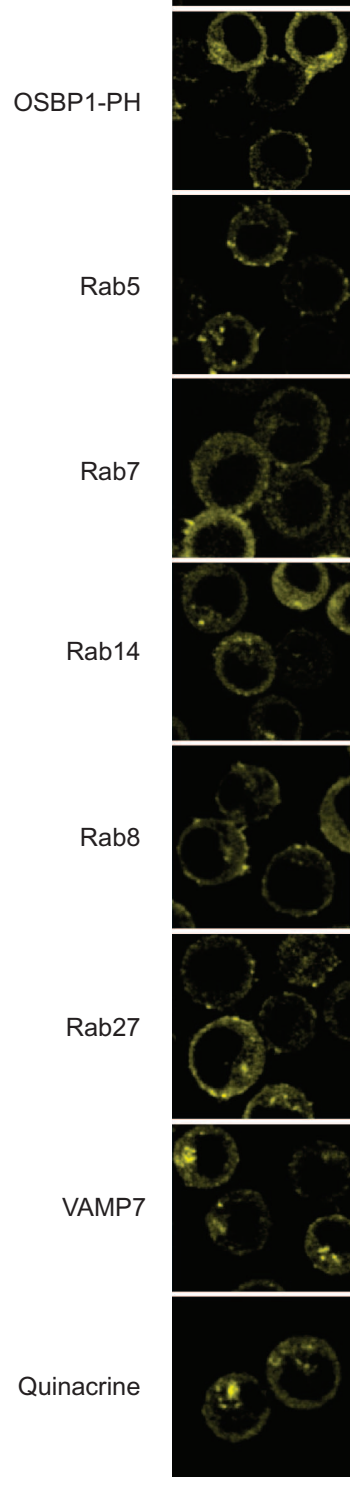
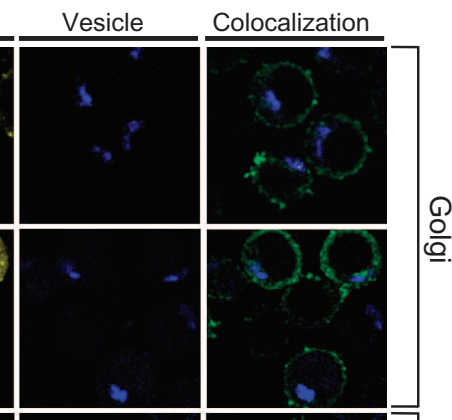

$\stackrel{\text { 을. }}{\underline{0}}$
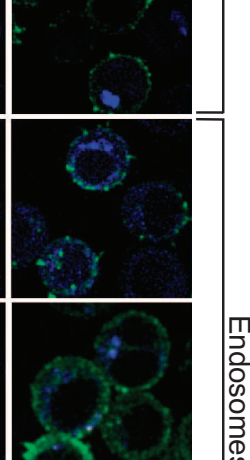

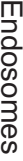
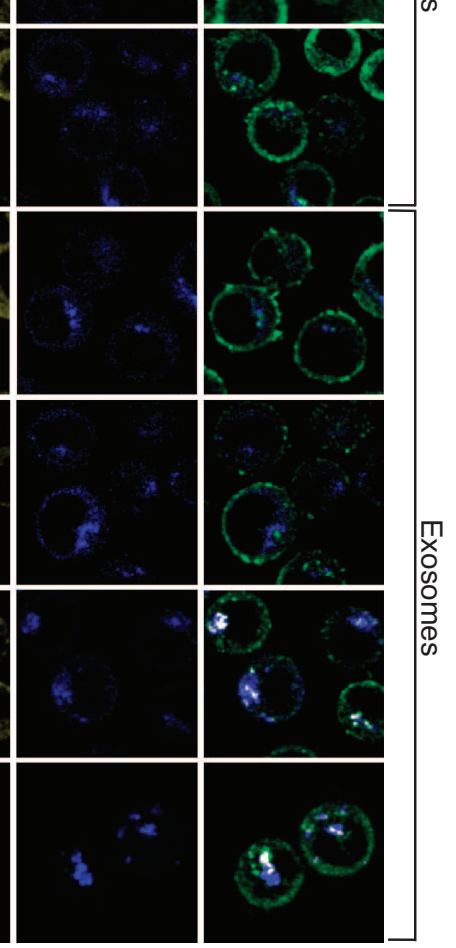

nature of the SLP-65-positive vesicles remain to be determined; however, they may function as a shuttle that delivers a preformed signaling cargo to sites of BCR activation at the plasma membrane.

\section{Anchoring SLP-65 to trans-Golgi vesicles initiates vesicular routing}

The microscopic characterization of the vesicular pool of SLP-65 together with the results of our floatation assays and NMR analysis suggested that the $\mathrm{N}$ terminus of SLP-65 directly targets the protein to exosome-like signaling organelles. Indeed, the $\triangle \mathrm{N}-\mathrm{SLP}-65$ mutant showed no colocalization with VAMP7-positive exosomes (Fig. 3, A and B). However, we pre-

B

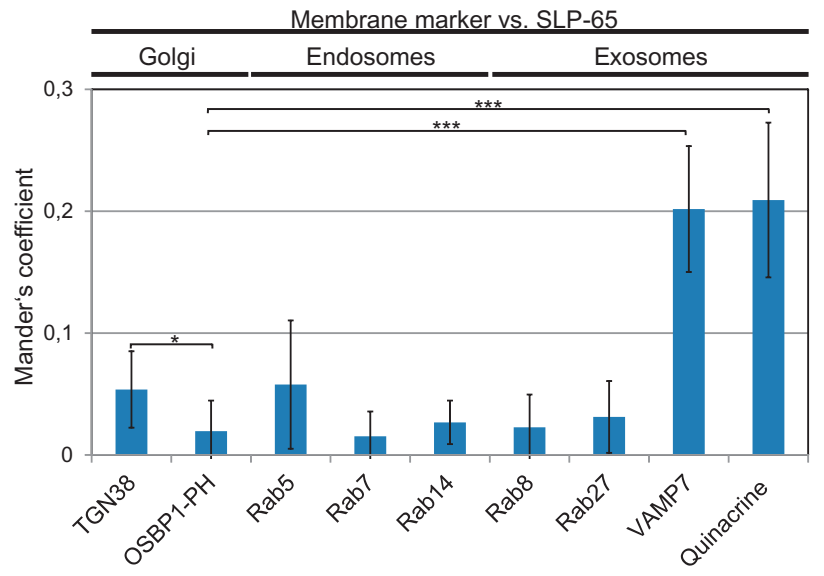

Fig. 2. SLP-65 colocalizes with VAMP7- and ATP-positive intracellular vesicles. (A) SLP-65-deficient DT40 cells were reconstituted to coexpress citrine-tagged SLP-65 (left), and the indicated cerulean-tagged vesicular markers (middle) were analyzed by confocal laser scanning microscopy to detect colocalization (right images). The colocalization images were generated after restrictive background subtraction with ImageJ software. Right: Noncolocalized citrine and cerulean signals are shown in green and blue, respectively, whereas colocalized signals are shown in a range from gray to white, with increasing degree of colocalization. (B) The degree of colocalization of cerulean signals with citrine signals was quantified by calculating the Mander's coefficient with ImageJ software. Values were determined from 5 to 18 independent experiments, with z-stacks of image sections containing 10 to 22 cells each, and are depicted as a bar diagram showing means \pm SD. Statistical significance was determined by a two-tailed unpaired $t$ test. ${ }^{\star} P<0.01 ;{ }^{\star \star *} P>0.0001$.

viously described that the SLP-65 binding partner CIN85 contributes to both the precise subcellular localization and the signaling function of the stable SLP-65-CIN85 complex (13). To assess the effect of CIN85 on the vesicular residence of SLP-65, we inactivated the proline-rich docking sites for CIN85 in SLP-65 and expressed the resulting SLP-65 mutant

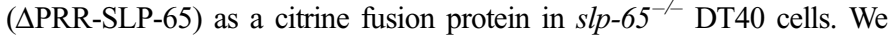
found that the loss of CIN85 binding, which is associated with SLP-65 signaling deficiency (13), resulted in SLP-65 becoming trapped in the trans-Golgi network compartment, as evidenced by its colocalization with the fluorescent fusion protein containing the PH domain of OSBP1 (Fig. 3, C and D). 
A

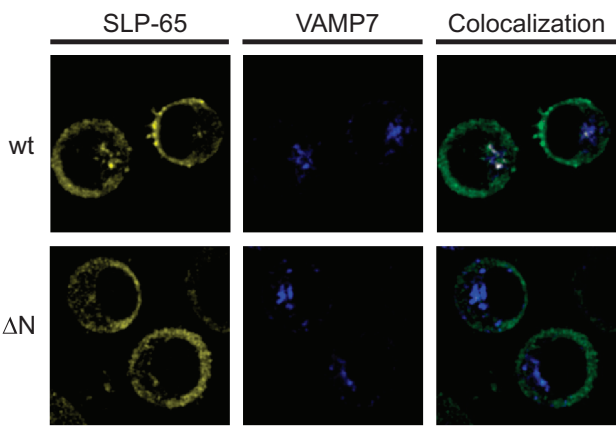

C
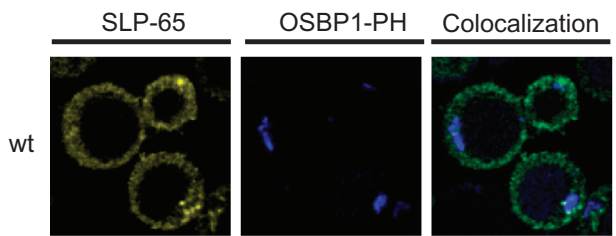

$\Delta \mathrm{N}$
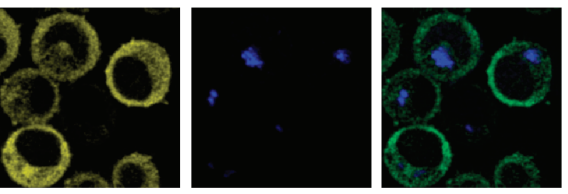

$\triangle \mathrm{PRR}$
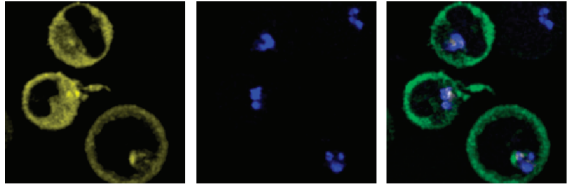

B

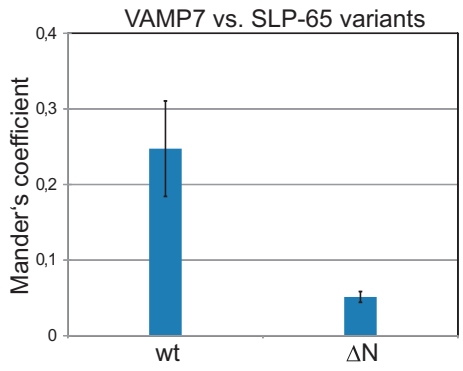

D

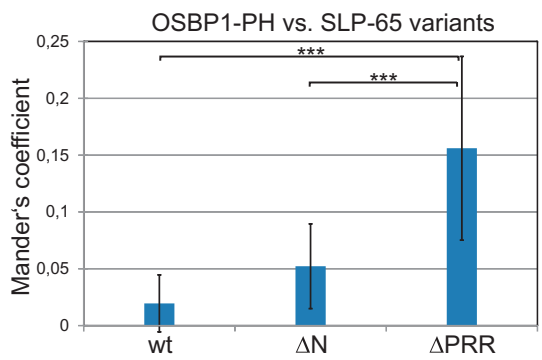

Fig. 3. The $\mathrm{N}$ terminus of SLP-65 is required for its colocalization with VAMP7-positive vesicles. (A) SLP-65-deficient DT40 cells reconstituted to coexpress citrine-tagged wt SLP-65 (top) or $\Delta \mathrm{N}-\mathrm{SLP}-65$ (bottom) together with a cerulean-VAMP7 fusion protein were analyzed by confocal laser scanning microscopy to detect colocalization. (B) Quantification of the extent of colocalization of citrine and cerulean signals in the cells represented in (A) was performed as described for Fig. 2B. Data are means \pm SD from four independent experiments, with z-stacks from 10 to 18 cells each. (C) DT40 B cells coexpressing a cerulean-tagged OSBP1-PH domain together with citrinetagged wt SLP-65 (top), $\Delta$ N-SLP-65 (middle), or $\triangle$ PRR-SLP-65 (bottom) were analyzed by confocal microscopy to detect colocalization. (D) Quantification of the extent of colocalization of citrine and cerulean signals in the cells represented in (C) was performed as described for Fig. 2B. Data are means \pm SD from 12 to 16 independent experiments, with z-stacks from 10 to 18 cells each. ${ }^{\star \star *} P>0.0001$.

This result suggests that there is cooperation between the $\mathrm{N}$ terminus of SLP-65 and CIN85 to tether SLP-65 to vesicular membranes with exosomal markers in resting $\mathrm{B}$ cells. It moreover suggested that the $\mathrm{N}$ terminus of SLP-65 provides a membrane anchor for Golgi-proximal vesicles. To directly test this hypothesis with a functional readout, we replaced the $\mathrm{N}$ terminus of wild-type SLP-65 or the $\triangle$ PRR-SLP-65 variant with the PH domain of the specific PIP4-binding protein OSBP1 (Fig. 4A), which associates with exquisite specificity to trans-Golgi membranes. Expression of the PH domain chimera of SLP-65 in slp-65 $5^{-/}$DT40 cells recapitulated the subcellular distribution of the OSBP1-PH domain (Fig. 4B). Although the OSBP1-PH domain did not target SLP-65 to VAMP7-positive exosomes (fig. S10), it reconstituted the BCR-triggered phosphorylation of SLP-65 and $\mathrm{Ca}^{2+}$ mobilization (Fig. 4, $\mathrm{C}$ and D). Thus, the $\mathrm{N}$ terminus of SLP-65 could be functionally replaced by a trans-Golgi-specific anchor under conditions of CIN85 binding. By contrast, the PH domain chimera of $\triangle$ PRR-SLP-65 mutant, which lacks CIN85-binding sites, only moderately supported BCR-dependent SLP-65 phosphorylation and $\mathrm{Ca}^{2+}$ mobilization (Fig. 4, C and D). On the basis of these findings, we suggest that precise vesicular tethering is primed by the $\mathrm{N}$ terminus of SLP-65 and then complemented by the constitutive SLP-65 binding partner CIN85.

\section{DISCUSSION}

The processing of signals emanating from the activated BCR relies on phosphorylation of the adaptor protein SLP65 by the tyrosine kinase Syk. It is well established that this process is indispensable for B cell activation and humoral immunity; however, the details of how and where Syk encounters its substrate SLP-65 were unclear. Here, we showed that in addition to undergoing classical protein-protein interactions, SLP-65 requires tethering at exosome-like vesicles for its phosphorylation and signaling. Our biochemical approaches and NMR spectroscopy showed that the $50 \mathrm{~N}$-terminal amino acid residues of SLP-65 acted as a direct membrane-anchoring device, which represents a previously uncharacterized type of lipid-binding domain. Furthermore, the N terminus of SLP-65 could be experimentally substituted by the OSBP1-PH domain, which does not bind to plasma membrane components, but constitutively recognizes PIP4 in postGolgi vesicles with exquisite specificity (15). First, this result excludes the possibility that the lipid-binding activity of the $\mathrm{N}$ terminus recruits cytoplasmic SLP65 to the inner leaflet of the plasma membrane in response to BCR stimulation. Instead, the $\mathrm{N}$ terminus mediates the assembly of SLP-65 complexes at vesicles already before the BCR is stimulated. Second, this result suggests that the $\mathrm{N}$ terminus alone is not sufficient to localize SLP-65 at exosome-like vesicles that contain VAMP7 and are detectable by quinacrine. Further evidence that additional elements are required for the vesicular localization of SLP-65 is provided by the ability of the N terminus of SLP- 65 to bind to more general components of cellular membranes. Indeed, colocalization of SLP-65 with exosomal markers required stable association with its binding partner CIN85. These data suggest that the N terminus of SLP65 and CIN85 cooperate, most likely in a sequential manner, for the subcellular targeting of the SLP-65-CIN85 signaling complex. The exact contribution of CIN85 remains to be elucidated; however, our data suggest that the presence of SLP-65 at vesicular compartments is a precondition for the sensing of BCR-derived signals and for subsequent B cell activation.

What is the biological advantage of the stimulation-independent, vesicular preassembly of SLP-65-CIN85 complexes for BCR signaling? A critical and common feature of signal transduction by cell surface receptors is the aspect of signal amplification. Syk easily accomplishes this task because its enzymatic activity suffices to phosphorylate a large quantity of downstream substrates despite its direct recruitment to the BCR with 
A

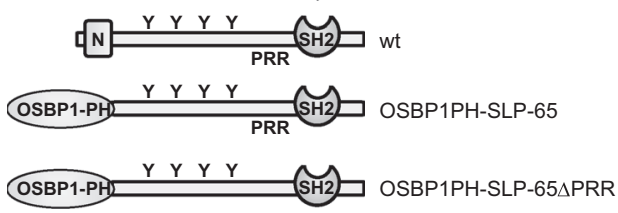

C

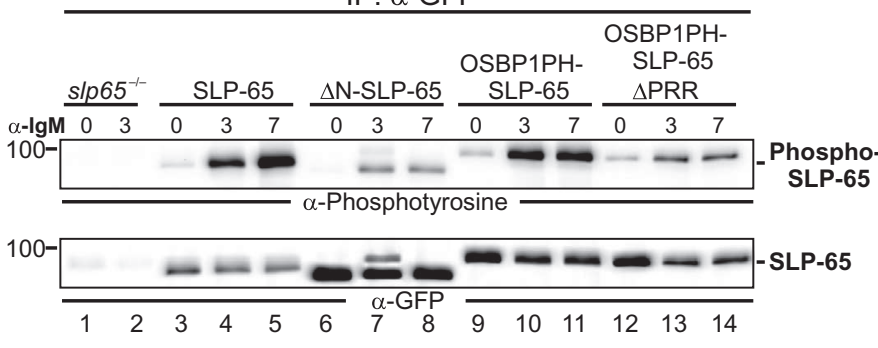

Fig. 4. The OSBP1-PH domain can functionally replace the $\mathrm{N}$ terminus of SLP-65. (A) Schematic representation of citrine-tagged SLP-65 constructs. Y, tyrosine phosphorylation site; PRR, proline-rich region. (B) SLP-65-deficient DT40 cells expressing the indicated citrine-tagged SLP-65 constructs were imaged by confocal microscopy. Images represent four independent experiments. (C) SLP-65-deficient DT40 cells used in (B) were left untreated or were treated with anti-IgM antibodies for the indicated times. Cells were

limited stoichiometry. By contrast, SLP-65 and CIN85 are inert adaptor proteins that cannot amplify the engagement of BCR signal effectors unless they oligomerize. Vesicular scaffolding of SLP-65, CIN85, and perhaps additional effectors provides a potent way to facilitate signal transmission by rapid, en masse delivery of a large, and perhaps complex, signaling cargo to the activated BCR. The necessary transport machinery might involve SNARE family proteins, such as VAMP7, which participates in intracellular trafficking and secretory pathways in several cell types (16), and which plays a role in the vesicular delivery of effector proteins to the $\mathrm{T}$ cell receptor complex $(18,19)$.

BCR-induced reorganization of the actin cytoskeleton (20) may actively or passively contribute to the process of SLP-65 recruitment by providing defined vesicle transport fibers or by releasing a cytoskeletal fence that in resting B cells prevents signaling vesicles from entering a cortical exclusion zone, respectively. In support of the later possibility, pharmacological disruption of the cytoskeletal network in B cells mimics BCR activation (20). In any case, our data argue against the common view that the initiation and processing of BCR signals rely solely on the sequential relocalization of individual signaling effectors to and at the plasma membrane. Instead, it appears that multimolecular effector complexes are preassembled at distinct signaling organelles already in the resting B cell, for which genetic evidence already existed (21).

An association with intracellular vesicles was reported for the $\zeta$ subunit of the T cell antigen receptor (TCR $\zeta)$, as well as for the kinase Lck and the adaptor protein linker of activated T cells (LAT), which are effectors downstream of the TCR $(18,19)$. In contrast to SLP-65, however, the membrane anchors of all three of these $\mathrm{T}$ cell signaling proteins are well characterized. Similar to other Src family kinase members, the protein tyrosine kinase Lck is attached to cellular membranes by its N-terminal
B

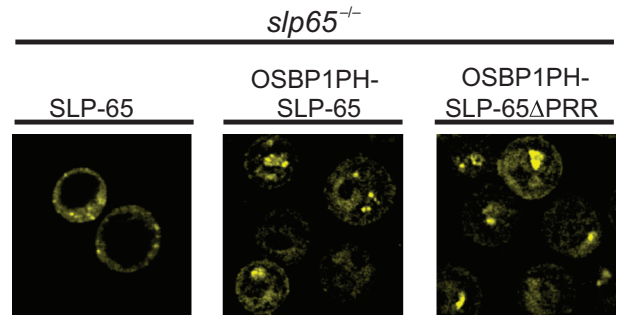

D

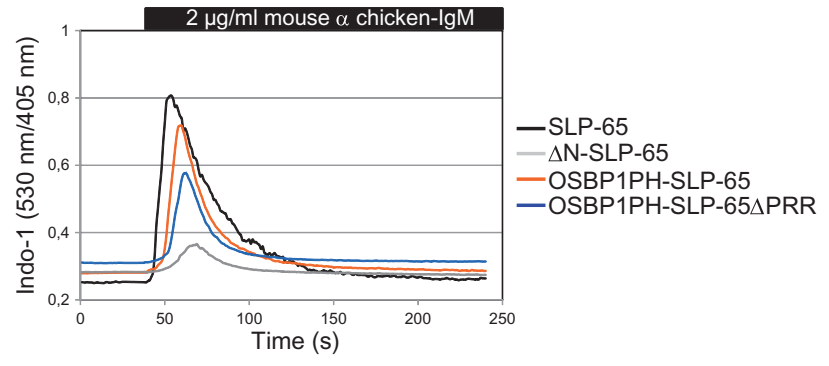

then lysed, subjected to immunoprecipitation with an anti-green fluorescent protein (GFP) antibody to pull down SLP-65 proteins, and analyzed by Western blotting with anti-phosphotyrosine (top) and anti-GFP (bottom) antibodies. Western blots are representative of three independent experiments. (D) SLP65-deficient DT40 cells expressing the indicated SLP-65 constructs were stimulated with anti-chicken IgM antibody and analyzed for $\mathrm{Ca}^{2+}$ mobilization by flow cytometry. Traces are representative of five independent experiments.

myristoyl anchor, whereas TCR $\zeta$ and LAT both have classical transmembrane regions. In spite of their more general mode of membrane anchoring, but similar to SLP-65, these T cell signaling elements are targeted to transport vesicles that contain exosomal markers, including VAMP7 $(18,19)$. The T cell paralog of SLP-65, SLP-76, is not directly associated with VAMP7-positive vesicles, which suggests that the intracellular localization of SLP-76 is mainly regulated by its binding partners, for example, LAT $(18,19)$. However, vesicular LAT may represent only a proportion of the total LAT involved in signaling (22), and the timing of the delivery of vesicular TCR effectors to the plasma membrane may lag behind that required for early TCR signaling (23). Nonetheless, the vesicular localization of $\mathrm{T}$ cell effectors was associated with productive TCR activation and the formation of a functional immunological synapse upon TCR ligation. Thus, a concept emerges, according to which the signaling competence of lymphocyte antigen receptors may be associated with the vesicular targeting and transport of their intracellular effector proteins. It will be interesting to investigate this concept in other receptor systems and to elucidate the inventory of SLP-65-positive signaling organelles.

\section{MATERIALS AND METHODS}

\section{Cell culture, microscopy, and signaling analyses}

DT40 cells were cultured, stimulated, and biochemically analyzed as described previously (24). Antibodies against GFP (Roche), phosphotyrosine (4G10, Millipore), SLP-65 (clone 2B11, BD Biosciences), and Akt (Cell Signaling Technology) were used for Western blotting analysis (at a 1:1000 dilution) and for immunoprecipitations ( 0.2 to $1 \mu \mathrm{g}$ per reaction). Ratiometric $\mathrm{Ca}^{2+}$ flux measurements were recorded by flow cytometry, as 
described previously (24). For live-cell confocal laser scanning microscopy (24), contrast, background subtraction, Gauss filtering, and colocalization analysis were applied with ImageJ software.

\section{Expression constructs, transfections, and retroviral gene transfer}

The complementary DNAs (cDNAs) encoding vesicular markers were amplified by reverse transcription polymerase chain reaction (RT-PCR) and subcloned into the expression plasmid pEGFP-N1 (BD Biosciences), in which the sequence encoding EGFP (enhanced green fluorescent protein) was replaced by that encoding cerulean, and subsequently cloned into pMSCV (BD Biosciences) containing a puromycin or bleomycin resistance cassette. The SLP-65-encoding constructs used were described previously (13). Point mutations were generated by site-directed mutagenesis with the QuikChange protocol (Stratagene). Constructs encoding chimeric proteins containing the OSBP1-PH domain (amino acid residues 87 to 189 ) and a SLP-65 variant lacking the $45 \mathrm{~N}$-terminal amino acids were generated by overlap extension PCR. DT40 B cells were transduced with retroviruses encoding SLP-65 proteins or vesicular marker proteins, as described previously (24), and transduced cells were selected with puromycin $(1 \mu \mathrm{g} / \mathrm{ml}$; InvivoGen) or bleomycin (70 $\mu \mathrm{g} / \mathrm{ml}$; Merck).

\section{Liposome floatation assays}

For an in vitro analysis of the ability of SLP-65 to bind to SUVs by liposome floatation, recombinant SLP-65 protein was generated. Escherichia coli (BL21) cells transformed with pET16b plasmid encoding SLP-65 were grown in $100 \mathrm{ml}$ of Luria broth medium containing ampicillin until they reached an $\mathrm{OD}_{600}$ (optical density at $600 \mathrm{~nm}$ ) of 0.6 . The $E$. coli were then incubated for 4 hours with $0.1 \mathrm{mM}$ IPTG (isopropyl- $\beta$-D-thiogalactopyranoside) to induce expression of SLP-65. Bacteria were suspended in LEW buffer [50 mM NaH${ }_{2} \mathrm{PO}_{4}(\mathrm{pH} 8.0), 300 \mathrm{mM} \mathrm{NaCl}$ ] and lysed by sonification. His-tagged SLP-65 was purified with Protino Ni-IDA columns (MachereyNagel) according to the manufacturer's instructions. Protein concentrations were measured with the Bradford assay. For liposome floatation, proteins were diluted in LEW buffer to a final concentration of $1 \mu \mathrm{M}$. SUV preparation and liposome floatation were performed as described previously (25), except that LEW buffer was used for SUV preparation and the Nycodenz gradient. SUVs were composed of $75 \%$ phosphatidylcholine and $25 \%$ phosphatidylethanolamine, of which $2 \%$ were labeled with Texas Red as tracer.

\section{Subcellular B cell fractionation}

DT40 cells were fractionated as previously described (26). Briefly, $3 \times 10^{8}$ cells were suspended in $250 \mathrm{mM}$ sucrose, $25 \mathrm{mM}$ Hepes, $20 \mathrm{mM} \mathrm{KCl}$, $2.5 \mathrm{mM} \mathrm{Mg}(\mathrm{OAc})_{2}$ and were disrupted by being passed 30 times through a Balch homogenizer. Upon sedimentation of nuclei, the supernatant was adjusted to a sucrose concentration of $55 \%$ and overlaid with $2 \mathrm{ml}$ of $45 \%$, $3 \mathrm{ml}$ of $35 \%$, and $3 \mathrm{ml}$ of $25 \%$ sucrose. Upon centrifugation for 5 hours at $217,000 \mathrm{~g}$, fractions were collected and analyzed by Western blotting.

\section{NMR spectroscopy}

A cDNA fragment encoding amino acid residues 5 to 50 of SLP- 65 was amplified by PCR and subcloned into a modified pET28a vector (Novagen). The uniformly ${ }^{13} \mathrm{C},{ }^{15} \mathrm{~N}$-labeled SLP-65 fragment was produced recombinantly in E. coli strain BL21(DE3) in minimal medium supplemented with ${ }^{13} \mathrm{C}$-glucose and ${ }^{15} \mathrm{~N}-\mathrm{NH}_{4} \mathrm{Cl}$ and was purified by immobilized metal affinity chromatography on Ni-NTA agarose (Qiagen). After proteolytic cleavage by tobacco etch virus (TEV) protease, the N-terminal His tag was removed by incubation with Ni-NTA agarose. After further purification by reversed-phase high-performance liquid chromatography, the SLP-65 frag- ment was dialyzed against NMR buffer [20 mM Hepes (pH 6.9), $150 \mathrm{mM}$ $\mathrm{KCl}$. Final protein concentrations were adjusted to $3.2 \mathrm{mM}$ in the sample for triple resonance experiments and to $0.1 \mathrm{mM}$ to test interactions with liposomes. The samples were subjected to HNCACB (27) and CBCAcoNH (28) experiments to determine the chemical shift assignments of $\mathrm{H}^{\mathrm{N}}, \mathrm{N}^{\mathrm{H}}, \mathrm{C}_{\alpha}$, and $\mathrm{C}_{\beta}$. HNCACB correlates the chemical shifts of $\mathrm{C}_{\beta i}$ and $\mathrm{C}_{\alpha i}$ in one frequency dimension with $\mathrm{N}^{\mathrm{H}}$ (second frequency dimension) and $\mathrm{H}^{\mathrm{N}}$ (third frequency dimension) of amino acid $i$ or $i+1$, whereas CBCAcoNH correlates the chemical shifts of $\mathrm{C}_{\beta i}$ and $\mathrm{C}_{\alpha i}$ in one frequency dimension through the carbonyl $(\mathrm{CO})$ with $\mathrm{N}^{\mathrm{H}}$ (second frequency dimension) and $\mathrm{H}^{\mathrm{N}}$ (third frequency dimension) of amino acid residue $i+$ 1. Side-chain assignments were obtained by analyzing hCCH-TOCSY (29), ${ }^{15} \mathrm{~N}-\mathrm{TOCSY}-\mathrm{HSQC}(30)$, and time-shared NOESY-HSQC (31). The HSQC experiment correlates a heteronucleus $\left({ }^{15} \mathrm{~N}\right.$ or $\left.{ }^{13} \mathrm{C}\right)$ in the first frequency dimension with the directly bound proton in the second frequency dimension. TOCSY (total correlation spectroscopy) correlates all nuclei of the same isotope that are present within a coupling network, whereas NOESY (nuclear Overhauser effect spectroscopy) correlates two protons that are close in space in two frequency dimensions. The randomcoil index was obtained as part of a TALOS-N calculation (32). The wateramide proton exchange rate was determined by CLEANEX-PM (33), with different mixing times of 5, 10, 25, and $50 \mathrm{~ms}$. Liposomes composed of $75 \%$ phosphatidylcholine and $25 \%$ phosphatidylethanolamine $(\mathrm{mol} / \mathrm{mol})$ were prepared by extensive sonication in NMR buffer at a total initial lipid concentration of $12.5 \mathrm{mM}$. From this solution, large multilamellar vesicles were removed by ultracentrifugation for 2 hours at $163,000 \mathrm{~g}$ at $25^{\circ} \mathrm{C}$. The supernatant, which consisted mostly of unilamellar vesicles, was subsequently used for NMR titrations.

\section{SUPPLEMENTARY MATERIALS}

www.sciencesignaling.org/cgi/content/full/7/339/ra79/DC1

Fig. S1. The N terminus of SLP-65 is essential for the BCR-dependent activation of ERK. Fig. S2. Differently tagged SLP-65 forms do not coimmunoprecipitate with each other.

Fig. S3. SLP-65 associates with membranes in resting human B cells.

Fig. S4. The L18K variant of SLP-65 cannot signal.

Fig. S5. The N terminus of SLP-65 is intrinsically disordered in solution and binds directly to liposomes.

Fig. S6. Inactivation of the putative leucine zipper motif only marginally affects BCR-induced $\mathrm{Ca}^{2+}$ mobilization.

Fig. S7. Lack of colocalization between SLP-65 and syntaxin 8 and SNAP23.

Fig. S8. N terminus-dependent attachment of SLP-65 to VAMP7-positive vesicles as revealed by bimolecular fluorescence complementation experiments.

Fig. S9. The vesicle-associated and cytosolic SLP-65 pools exchange rapidly with each other.

Fig. S10. The OSBP1-PH domain is insufficient to target chimeric SLP-65 protein to VAMP7-positive vesicles.

\section{REFERENCES AND NOTES}

1. B. J. Mayer, R. Gupta, Functions of $\mathrm{SH} 2$ and $\mathrm{SH} 3$ domains. Curr. Top. Microbiol. Immunol. 228, 1-22 (1998)

2. M. Reth, J. Wienands, Initiation and processing of signals from the B cell antigen receptor. Annu. Rev. Immunol. 15, 453-479 (1997).

3. J. Wienands, J. Schweikert, B. Wollscheid, H. Jumaa, P. J. Nielsen, M. Reth, SLP-65: A new signaling component in $B$ lymphocytes which requires expression of the antigen receptor for phosphorylation. J. Exp. Med. 188, 791-795 (1998).

4. C. Fu, C. W. Turck, T. Kurosaki, A. C. Chan, BLNK: A central linker protein in B cell activation. Immunity 9, 93-103 (1998).

5. N. Engels, B. Wollscheid, J. Wienands, Association of SLP-65/BLNK with the B cell antigen receptor through a non-ITAM tyrosine of Ig- $\alpha$. Eur. J. Immunol. 31, 2126-2134 (2001)

6. S. Kabak, B. J. Skaggs, M. R. Gold, M. Affolter, K. L. West, M. S. Foster, K. Siemasko, A. C. Chan, R. Aebersold, M. R. Clark, The direct recruitment of BLNK to immunoglobulin $\alpha$ couples the B-cell antigen receptor to distal signaling pathways. Mol. Cell. Biol. 22, 2524-2535 (2002). 
7. G. A. Koretzky, F. Abtahian, M. A. Silverman, SLP76 and SLP65: Complex regulation of signalling in lymphocytes and beyond. Nat. Rev. Immunol. 6, 67-78 (2006).

8. J. Wienands, Signal transduction elements of the B cell antigen receptor and their role in immunodeficiencies. Immunobiology 202, 120-133 (2000).

9. Y. Kulathu, E. Hobeika, G. Turchinovich, M. Reth, The kinase Syk as an adaptor controlling sustained calcium signalling and B-cell development. EMBO J. 27 1333-1344 (2008).

10. K. Kometani, T. Yamada, Y. Sasaki, T. Yokosuka, T. Saito, K. Rajewski, M. Ishiai, M. Hikida, T. Kurosaki, CIN85 drives B cell responses by linking BCR signals to the canonical NF-кB pathway. J. Exp. Med. 208, 1447-1457 (2011).

11. F. Köhler, B. Storch, Y. Kulathu, S. Herzog, S. Kuppig, M. Reth, H. Jumaa, A leucine zipper in the $\mathrm{N}$ terminus confers membrane association to SLP-65. Nat. Immunol. 6, 204-210 (2005)

12. A. Abudula, A. Grabbe, M. Brechmann, C. Polaschegg, N. Herrmann, I. Goldbeck K. Dittmann, J. Wienands, SLP-65 signal transduction requires Src homology 2 domain-mediated membrane anchoring and a kinase-independent adaptor function of Syk. J. Biol. Chem. 282, 29059-29066 (2007).

13. T. Oellerich, V. Bremes, K. Neumann, H. Bohnenberger, K. Dittmann, H. H. Hsiao, M. Engelke, T. Schnyder, F. D. Batista, H. Urlaub, J. Wienands, The B-cell antigen receptor signals through a preformed transducer module of SLP65 and CIN85. EMBO J. 30, 3620-3634 (2011).

14. M. V. Berjanskii, D. S. Wishart, A simple method to predict protein flexibility using secondary chemical shifts. J. Am. Chem. Soc. 127, 14970-14971 (2005).

15. A. Balla, G. Tuymetova, A. Tsiomenko, P. Várnai, T. Balla, A plasma membrane poo of phosphatidylinositol 4-phosphate is generated by phosphatidylinositol 4-kinase type-III alpha: Studies with the $\mathrm{PH}$ domains of the oxysterol binding protein and FAPP1. Mol. Biol. Cell 16, 1282-1295 (2005).

16. M. Chaineau, L. Danglot, T. Galli, Multiple roles of the vesicular-SNARE TI-VAMP in post-Golgi and endosomal trafficking. FEBS Lett. 583, 3817-3826 (2009).

17. G. G. Yegutkin, A. Mikhailov, S. S. Samburski, S. Jalkanen, The detection of micromolar pericellular ATP pool on lymphocyte surface by using lymphoid ecto-adenylate kinase as intrinsic ATP sensor. Mol. Biol. Cell 17, 3378-3385 (2006).

18. P. Larghi, D. J. Williamson, J. M. Carpier, S. Dogniaux, K. Chemin, A. Bohineust, L. Danglot, K. Gaus, T. Galli, C. Hivroz, VAMP7 controls T cell activation by regulating the recruitment and phosphorylation of vesicular Lat at TCR-activation sites. Nat. Immunol. 14, 723-731 (2013).

19. H. Soares, R. Henriques, M. Sachse, L. Ventimiglia, M. A. Alonso, C. Zimmer, M. I. Thoulouze, A. Alcover, Regulated vesicle fusion generates signaling nanoterritories that control $\mathrm{T}$ cell activation at the immunological synapse. J. Exp. Med. 210, 2415-2433 (2013).

20. B. Treanor, D. Depoil, A. Bruckbauer, F. D. Batista, Dynamic cortical actin remodeling by ERM proteins controls BCR microcluster organization and integrity. J. Exp. Med. 208, 1055-1068 (2011).

21. J. Wienands, O. Larbolette, M. Reth, Evidence for a preformed transducer complex organized by the B cell antigen receptor. Proc. Natl. Acad. Sci. U.S.A. 93, 7865-7870 (1996).

22. M. A. Purbhoo, H. Liu, S. Oddos, D. M. Owen, M. A. Neil, S. V. Pageon, P. M. French C. E. Rudd, D. M. Davis, Dynamics of subsynaptic vesicles and surface microclusters at the immunological synapse. Sci. Signal. 3, ra36 (2010).

23. L. Balagopalan, V. A. Barr, R. L. Kortum, A. K. Park, L. E. Samelson, Cutting edge: Cell surface linker for activation of T cells is recruited to microclusters and is active in signaling. J. Immunol. 190, 3849-3853 (2013).

24. B. Stork, K. Neumann, I. Goldbeck, S. Alers, T. Kähne, M. Naumann, M. Engelke, J. Wienands, Subcellular localization of Grb2 by the adaptor protein Dok-3 restricts the intensity of $\mathrm{Ca}^{2}$ signaling in B cells. EMBO J. 26, 1140-1149 (2007).
25. R. A. Busse, A. Scacioc, J. M. Hernandez, R. Krick, M. Stephan, A. Janshoff, M. Thumm, K. Kühnel, Qualitative and quantitative characterization of protein-phosphoinositide interactions with liposome-based methods. Autophagy 9, 770-777 (2013)

26. I. Bethani, T. Lang, U. Geumann, J. J. Sieber, R. Jahn, S. O. Rizzoli, The specificity of SNARE pairing in biological membranes is mediated by both proof-reading and spatia segregation. EMBO J. 26, 3981-3992 (2007)

27. M. Wittekind, W. J. Metzler, L. Mueller, Selective correlations of amide groups to glycine alpha-protons in proteins. J. Magn. Reson. B 101, 214-217 (1993)

28. S. Grzesiek, A. Bax, Correlating backbone amide and side-chain resonances in larger proteins by multiple relayed triple resonance NMR. J. Am. Chem. Soc. 114, 6291-6293 (1992).

29. A. Bax, G. M. Clore, A. M. Gronenborn, $\mathrm{H}-1-\mathrm{H}-1$ correlation via isotropic mixing of $\mathrm{C}-13$ magnetization, a new 3-dimensional approach for assigning $\mathrm{H}-1$ and $\mathrm{C}-13$ spectra of C-13-enriched proteins. J. Magn. Reson. 88, 425-431 (1990).

30. D. Marion, P. C. Driscoll, L. E. Kay, P. T. Wingfield, A. Bax, A. M. Gronenborn, G. M. Clore, Overcoming the overlap problem in the assignment of proton NMR spectra of large proteins by use of three-dimensional heteronuclear proton-nitrogen-15 HartmannHahn-multiple quantum coherence and nuclear Overhauser-multiple quantum coherence spectroscopy: Application to interleukin 1.beta. Biochemistry 28, 6150-6156 (1989)

31. M. Sattler, M. Maurer, J. Schleucher, C. Griesinger, A simultaneous ${ }^{15} \mathrm{~N},{ }^{1} \mathrm{H}$ and ${ }^{13} \mathrm{C},{ }^{1} \mathrm{H}-\mathrm{HSQC}$ with sensitivity enhancement and a heteronuclear gradient echo. J. Biomol. NMR 5, 97-102 (1995).

32. Y. Shen, A. Bax, Protein backbone and sidechain torsion angles predicted from NMR chemical shifts using artificial neural networks. J. Biomol. NMR 56, 227-241 (2013).

33. T. L. Hwang, P. C. van Zijl, S. Mori, Accurate quantitation of water-amide proton exchange rates using the phase-modulated CLEAN chemical EXchange (CLEANEX-PM) approach with a Fast-HSQC (FHSQC) detection scheme. J. Biomol. NMR 11, 221-226 (1998).

Acknowledgments: We thank I. Heine and C. Schwiegk for excellent technical assistance; O. Babosova, A.-S. Ernst, T. Bäumler, S. Torke, and A. Scacioc for generating various data and reagents; and M. E. Guimaraes de Araujo, L. Huber, and K. Kühnel for their help with setting up the nitrogen cavitation and liposome floatation assays. We are gratefu to L. Fonseca for discussion and A. Woehler for help with the FRAP experiments. Funding: This work was supported by the Deutsche Forschungsgemeinschaft through SFB 860 (project B5), TRR130 (project 08), and the Max Planck Society. Author contributions: M.E. designed and performed biochemical and imaging experiments; S.P. and J.K investigated the vesicular association of SLP-65 in vitro and in vivo, respectively, and they analyzed SLP-65 signaling mutants, with contributions by J.B.; L.W. assigned the $\mathrm{N}$ terminus and analyzed the liposome binding of SLP-65 by NMR spectroscopy; N.H. provided expression constructs; S.B. produced the recombinant protein samples for NMR; C.G. supervised the NMR studies and NMR data analyses; and J.W. supervised the project and wrote the paper. Competing interests: The authors declare that they have no competing interests.

\section{Submitted 22 January 2014}

Accepted 1 August 2014

Final Publication 19 August 2014

10.1126/scitranslmed.2005104

Citation: M. Engelke, S. Pirkuliyeva, J. Kühn, L. Wong, J. Boyken, N. Herrmann, S. Becker, C. Griesinger, J. Wienands, Macromolecular assembly of the adaptor SLP-65 at intracellular vesicles in resting B cells. Sci. Signal. 7, ra79 (2014). 